\section{EL CONSERVADOR Y SU PROBLEMÁTICA}

La entrada del Restaurador español en el museo supuso inicialmente el traspaso del Restaurador de la Colección Real a las primeras Colecciones públicas; aquellos museos-depósitos, nacidos de las desamortizaciones, escasamente daban cabida a la figura del Conservador Restaurador y finalmente la propia dinámica del museo decimonónico español y la atención a los "Monumentos" explicaban su indefinición. Durante el primer tercio del siglo XX la aparición de los primeros Talleres de Restauración, bien en el Museo del Prado o en el Museo Arqueológico Nacional, producía el acceso del Conservador Restaurador a la Administración Pública mediante el sistema de oposición y quedaban patentes las distintas especialidades. El nacimiento del Servicio de Restauración y Conservación de obras de provincia traía también como consecuencia la creación del Cuerpo de Restauradores y Forradores.

La falta de desarrollo cualitativo de los museos españoles y su escasez de personal durante el segundo tercio de siglo muestra al fin del mismo a la figura del Restaurador en una situación problemática, escasamente representada en los museos españoles y con problemas de titulación.

Esta es la herencia que recibe el Restaurador en los finales del siglo $X X$, una indefinición, de la que se han hecho eco las críticas, bien referidas al personal del Museo en general, bien aquellas específicas sobre su figura.

En el último tercio de siglo los museos españoles muestran a la figura del restaurador en una situación problemática, escasamente representada y con problemas de titulación.
Amplias son las críticas de Aurora León acerca de este tema en 1978' y de Luis Caballero Zoreda, en 1982, sobre la falta de personal profesionalizado como "probablemente el más grave problema... de todos los Museos españoles"2. Específicas las que expresa Ignacio Gárate Rojas señalando "el ridículo número de restauradores contratados por el Estado y nada digamos de la Iglesia u otros poseedores de nuestro Patrimonio"3.

En 1973 el estudio del panorama de los Museos españoles realizado por Gratiano Nieto Gallo, hace una síntesis centrada en la situación de los Restauradores:

"Otro grupo de técnicos lo integran los restauradores, colaboradores de singular importancia también, pero con los que cuentan unos pocos Museos tan sólo. Hasta la fecha, los pocos técnicos de esta cla- se con que algunos museos cuentan, excepción hecha de alguno que es titulado en Bellas Artes, tienen una formación empírica, conseguida tras muchos años de práctica, gracias a lo cual han logrado dominar las técnicas necesarias para los diferentes sectores en que trabajan, con eficacia sin duda, pero sin estar al día en cuanto a criterios y sistemas modernos de tratamientos, a pesar de lo cual, la labor que han realizado sólo elogios merece, pues gracias a su intervención han podido salvarse muchas obras de especial significación"4.

Este autor señala puntos problemáticos a los que se añaden otros contemplados en diferentes textos:

\section{La escasez numérica.}

Son pocos los datos concretos conocidos sobre el número de restauradores de los museos españoles, sin embargo el análisis de la plantilla de personal de los museos más representativos y las referencias recogidas en los estudios del panorama museográfico español apuntan con unanimidad ${ }^{5}$.

Por ejemplo, el Museo Arqueológico Nacional en el año 198 I contaba con una Unidad de Restauración, con el jefe de la Unidad perteneciente al Cuerpo de Ayudantes de Archivos, Bibliotecas y Museos y seis Restauradores, cuatro de la categoría Restaurador interino, no escalafonado y dos Restauradores contratados, no escalafonados. Cuatro de las plazas pertenecen a la Administración del Estado, la de Restauradores interinos y las otras son contratos laborales del Patronato Nacional de Museos, con la consideración de Ayudantes de Restauración; de éstos uno de ellos actúa como vigilante nocturno, los demás como obreros 6 .

La plantilla teórica del Museo Nacional del Prado en 1985 contaba con dos plazas no escalafonadas del grupo D, dentro de la categoría funcionarios de la Administración Civil y de la categoría personal contratados en Régimen laboral, dos titulados de Grado Medio Restauración y un Restaurador?

\section{La falta de homogeneidad administrativa.}

Al reducido número de Restauradores en los Museos, se debe añadir la falta de homogeneidad administrativa: Restaurador interino, funcionario de la administración civil, plaza no escalafonada, grupo $D$, restaurador contratado en Régimen laboral dependiente del Patronato Nacional de Museos ${ }^{8}$.

La falta de exigencia de cualificación o titulación específica.

Los Museos españoles tienen Restauradores a los que no se les exige una formación, cualificación o titulación específica hasta 1969, fecha en la cual se exige para trabajar en un centro público el título de Restaurador. La existencia de la titulación de diplo- 
mado expedida por las Escuelas de Bellas Artes no implicaba en la Administración Civil la contemplación de esta como requisito necesario para cubrir plaza de Restaurador presentándose, excepcionalmente, el titulado por Bellas Artes. Incide en el tema la existencia de Restauradores con formación empírica, sin criterios y faltos de conocimientos en tratamientos modernos.

El texto de Gratiano Nieto Gallo expone que a excepción de alguno que es titulado en Bellas Artes, el restaurador tiene formación empírica, conseguida tras años de práctica. Una vez más la crítica y la valoración del restaurador, como ocurre en otros periodos de la historia de la profesión, se plantea la ecuación perfil-formación.

Francisco de Goya, Vicente Poleró y Toledo y después Claudio Boutelou habían referido esta cuestión, el primero apuntando la necesidad de sólidos principios, el segundo aportando una reflexión sobre ser Restaurador y serlo sin estar respaldado por una experiencia, dotes y unos conocimientos específicos y el último haciendo alusión a la especialización.

En este periodo, la relación restaurador-formación es un problema que también se plantea. Dice Ignacio Gárate Rojas: "Para poder ejercer una acción adecuada hay que formar técnicos. Hay titulados, es cierto y aunque no tantos como los necesarios, la gran mayoría está en paro. Han hecho unos estudios cortos, pero un buen restaurador necesita de diez a doce años de continua práctica debidamente dirigida, para que empiece a dar frutos. Poco dice un título, pues igual que citábamos el largo proceso del artista en su creación, para familiarizarse con las técnicas y materias, no será menos el esfuerzo y la práctica del que va a actuar sobre esa Obra de Arte, para detener su proceso destructor",

\section{La ausencia de un Cuerpo Técnico de} Restauradores y de oposiciones.

Luis Caballero en 1982 recoge este tema en su análisis sobre los Restauradores del Museo Arqueológico Nacional de Madrid, bajo el epígrafe "Problemas de Restauradores": "Como se sabe, los Restauradores deberían estar agrupados en un Cuerpo Técnico, creado e incluso convocadas en varias ocasiones las oposiciones que no llegaron a cubrirse. Ello provoca un problema de estabilidad especial a los Restauradores, profundamente descontentos, y con razón en el tema. A nivel anecdótico obsérvese que cuando el que redacta terminó sus estudios profesionales conoció a varios restauradores que hoy son interinos en el Museo y que entonces se encontraban en su misma situación, recién salidos de la Escuela de Restauración. Los reiterados intentos de solucionar su estado, el último en trámite, no han dado, aún, resultado positivo"I0.

La problemática del Restaurador puede por tanto ser sintetizada: falta de homogeneidad administrati- va, escasez numérica, ausencia de oposiciones, ausencia de un Cuerpo Técnico de Restauradores y con problemas en cuanto a titulaciones.

Baste señalar la carencia de trabajos e investigaciones sobre este tema. El único conocido fue llevado a cabo por M Ma Fernanda Morón de Castro en 1992 con respecto al Restaurador en los Museos andaluces y por su excepcionalidad, quedan recogidas aquí sus conclusiones, que apuntan además de los temas señalados un nuevo punto: la problemática de las titulaciones.

“...la precaria situación del Conservador-Restaurador en el panorama museológico andaluz. La administración autonómica parece no tener conciencia de ello. Como soluciones a este problema habría que modificar la relación y categoría de puestos de trabajo, considerando al restaurador en el nivel de licenciado y no sólo en el de diplomado. Se podrían dotar las plazas de restauradores y ayudantes de restauradores en los museos con carácter de funcionario, a las que se accederían por concurso oposición"l!.

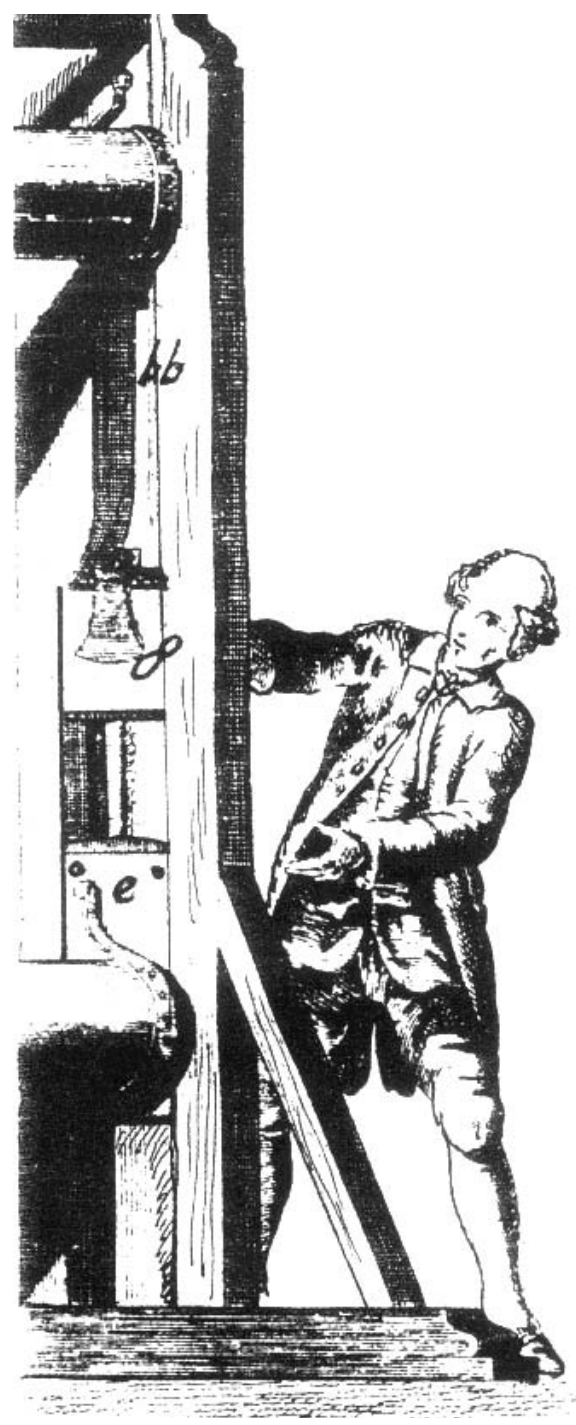

NOTAS

(1) "La ampliación del equipo es necesidad urgente de acometer, dotando a cada sección o departamento del personal necesario para el desarrollo dinámico de sus funciones. Abaratar los desembolsos del Museo con la reducción del personal es no sólo perjudicial para la calidad del trabajo,... y antieconómico a la larga, pues la falta de atención a las obras produce consecuencias de mayores costos económicos, intelectuales y técnicos". LEÓN, A. El Museo. Teoría, praxis y utopía, Madrid 1978, pág. 331.

(2) CABALLERO ZOREDA, LUIS. Funciones, Organización y Servicios de un Museo: El Museo Arqueologico Nacional de Madrid. Asociación Española de Archiveros, Bibliotecarios, Museólogos y Documentalistas, Guadalajara, 1982, pág. 134.

(3) GARATE ROJAS, I."Un llanto por nuestro Patrimonio". Análisis e investigaciones Culturales. Museos, núm. 22, enero-marzo, 1985, pág. 93.

(4) NIETO GALLO, G. Panorama de los Museos Españoles y cuestiones Museológicas. Asociación Nacional de Bibliotecarios, Archiveros y Arqueólogos, Madrid, 1973, pág 75.

(5) Sería interesante tener datos concretos, sobre la cantidad de Restauradores que forman la plantilla de los Museos de titularidad estatal y en general de todo el Sistema Español de Museos, a través del Ministerio de Cultura. El estudio de los Museos más significativos y las escasas pero interesantes alusiones que sobre el tema existen en los estudios del panorama museográfico español, resultan, suficientes y reveladores. 


\section{El problema de las titulaciones.}

En 1969 un cambio importante viene a señalar la trayectoria del Conservador Restaurador: la necesidad de titulación para cubrir plazas convocadas por oposición en los museos. El cambio tiene raíces profundas, desde aquellas relativas al personal del museo, a aquellas relativas a los funcionarios de la administración pública y las transformaciones que derivan en el nacimiento de los nuevos perfiles profesionales.

La antigua relación entre el Museo Nacional de Pinturas y Escultura y la Escuela de Pintura y Escultura de Madrid para cubrir por oposición una plaza de Restaurador en el primer Museo español en 1922'2, cambia en estas fechas cuando, el sistema de oposición define el contenido de la misma y el perfil del nuevo Conservador Restaurador.

Y si, a principios de siglo, el Museo se remitía a la Escuela de Pintura y Escultura ahora se dirige a la Escuela de Artes Aplicadas, a la Restauración creada recientemente en Madrid. La exigencia de poseer un título para el ejercicio de la profesión de Restaurador había quedado recogido en su Reglamento, realizado en 1969, concretamente en su art. $3^{\circ}$, donde se expone la necesidad de poseer el título de Restaurador en la sección correspondiente, expedido por el Ministerio, de la Escuela de Artes Aplicadas o el diploma de Restaurador que se expide en las Escuelas Superiores de Bellas Artes, para participar en las oposiciones que se convoquen para cubrir plazas de este carácter en los Centros del Estado.

La situación laboral y profesional, pobre, poco homogénea y por contrato, el sistema de acceso, sin cualificación específica y sin exigir la diplomatura, hasta entonces expedida por las Escuelas de Bellas Artes, sufre un cambio importante: se exige la titulación para el cargo de Restaurador de Museo.

Esta cuestión tuvo un efecto inmediato, creando inquietud en los propios restauradores que venían desempeñando su trabajo en los museos.

Las disposiciones adicionales del Reglamento de la Escuela de Artes Aplicadas a la Restauración, contemplaba sin embargo el caso, especificándose que quedaban exceptuados y dispensados por el Ministerio de este requisito, quienes a la entrada en vigor de la orden se hallasen desempeñando plazas de Restaurador en Centros del Estado, con nombramiento expedido por el Ministerio de Educación y Ciencia y las personalidades de notorio prestigio en la materia, previo informe de la Real Academia de San Fernando.

Fue una disposición mediadora entre el pasado y el presente. Los Museos estaban ocupados por Restauradores nombrados por el Ministerio directamente o bien mediante la vía del mérito a través de la Real Academia.
Las oposiciones al cargo de Restaurador de Museos remitían al primer tercio de siglo y se había detenido posteriormente pasando por un periodo poco profesionalizado' ${ }^{3}$, desde 1934 hasta 1968, en el que se transforma el sistema antiguo, se crea el Ministerio de Educación y el propio Instituto Central de Conservación y Restauración.

En 197| los Restauradores de Museos que venían desempeñando el cargo solicitan al Ministerio de Educación y Ciencia acogerse a la disposición transitoria primera de la mencionada Orden de Regulación de la Escuela y se establece una prueba de reválida de conjunto para obtener el título académico de Restaurador por la citada Escuela. Esta organiza un curso abreviado de "Historia del Arte, Arqueología, Química aplicada a la Restauración y criterios de Conservación y Restauración" previo a la prueba de reválida al que pudieron asistir los Restauradores antes mencionados ${ }^{14}$.

El examen se realiza sobre las asignaturas que componen el Plan de estudios de la citada Escuela y con ello quedaba establecido desde la Administración el perfil del nuevo Conservador Restaurador.

Estos son los ejercicios de que constó la prueba: a) Descripción, clasificación razonada y datación de una pieza arqueológica o etnológica (Sección de Restauración de Objetos Arqueológicos o Etnológicos) y clasificación estilística de una obra (Sección Restaurador de Obras de Arte).

b) Informe técnico sobre su estado de conservación.

c) Informe técnico del procedimiento de restauración a seguir.

d) Medios auxiliares que pueden intervenir en la Conservación y Restauración de una obra, especificándose aquellas que deben aplicarse en un caso concreto.

e) Descripción de la técnica de ejecución de la obra en estudio.

f) Ejercicio práctico.

En el año 1974 se aprueba el texto para la redacción del título académico de los Restauradores de Obras de Arte u Objetos arqueológicos o etnológicos y en 1978 el Ministerio de Educación y Ciencia accede a realizar de nuevo la prueba a los solicitantes $^{15}$.

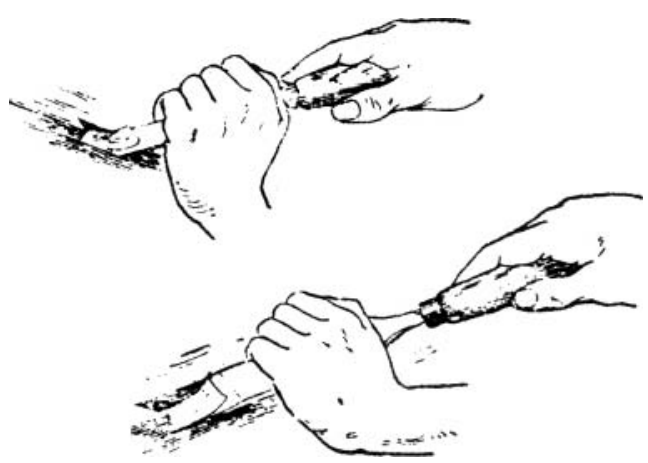

\footnotetext{
9) GARTA ROJAS I. "Un llant

nuestro Patrimonio", Análisis investigaciones culturales, Museos, núm. 22, enero-marzo, 1985, pág. 93.

de un Museo. El Museo Arqueológi-

administrativa el Restaurador de 23 de septiemeto 1.635

de Restaurador expedido por las Restauración en la tarifa VI, D, como asimilado a los títulos y Clase VI, D, dispuesta en 1959 permanece vigente en nuestros días.
} 
En la introducción de la citada orden se expone que la petición de un nuevo examen se fundamenta en que vienen desempeñando sus funciones en Centros dependientes del Estado, Diputaciones y Ayuntamientos, a satisfacción de los correspondientes centros, con una antigüedad superior a diez años de trabajo de la especialidad, el que existe el precedente de la anterior prueba y la imposibilidad por circunstancias tanto personales como de trabajo de desplazarse a Madrid, en donde radica la única Escuela de Artes Aplicadas a la Restauración, para cursar en la misma los estudios regulares para la obtención de la correspondiente titulación académica".

Aunque es cierto que la única Escuela de Artes Aplicadas a la Restauración radicaba en Madrid, también lo es que el diploma de Restaurador, expedido por las Escuelas de Bellas Artes parecía no existir.

A través de estos acontecimientos quedaban unificados los Restauradores de los museos españoles bajo la nueva titulación, se unificaba el perfil del Conservador Restaurador con respecto a la titulación, a la vez que nacía el problema de la existencia de una doble titulación.

El Restaurador era reconocido Diplomado en la clase $\mathrm{VI}, \mathrm{D}^{16}$. La posterior transformación de la Escuela de Bellas Artes en Facultad traería consigo la Licenciatura de Bellas Artes, especialidad Restauración y Conservación de Obras de Arte, sin embargo no encontraba eco en los organismos de la Administración. Académicamente existía doble titulación, el título de la Escuela y la Licenciatura de la Facultad, administrativamente sin embargo, ambas eran reconocidas con la Clasificación VI, D.

En resumen, llevado a cabo el proceso de reciclaje del perfil del Restaurador de Museos, del antiguo sistema al nuevo, todo Restaurador de Museos de España disfrutaba de titulación y parecía partir de nuevo de un punto cero, con un perfil, cuyo diseño había sido trazado por el Ministerio a través de la Escuela de Conservación y Restauración.

Para terminar es lícito establecer un paralelismo entre aquel borrador de 1922 y la propuesta de oposición de 1978, porque conocido su contexto y la evolución, aporta una idea reveladora.
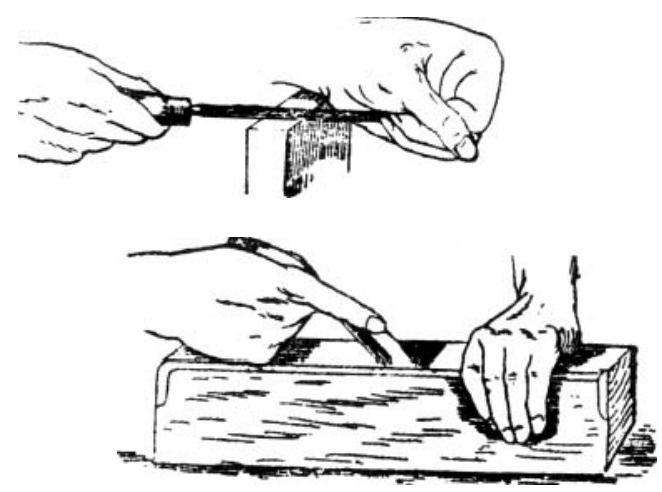

Desde aquella oposición propuesta por la Escuela de Pintura y Escultura a esta otra prueba establecida por la Escuela de Procedimientos, Arte de la Restauración, y Museología, median muchos años (1922-1978) sin embargo se unen porque entre ellas habían existido escasas oposiciones para cubrir plazas de Restaurador en los Museos, Centros dependientes del Estado, en Diputaciones o Ayuntamientos.

Entre los contenidos de una y otra existen lógicamente grandes diferencias, mientras que en 1922 , se planteaba dibujar una figura del antiguo, pintar del natural una figura y ropajes, proceder a una limpieza o escribir una disertación pequeña sobre el tema referente a la plaza y responder a cinco preguntas sacadas a suerte; en 1978 se establece la diversificación y especialización de la profesión (Arqueología, Etnología y Obra de Arte), se considera el estudio previo de la obra desde un punto de vista descriptivo, su clasificación y estudio; contempla el informe técnico del estado de conservación y el informe de restauración; el estudio técnico de la obra; los instrumentos auxiliares para la Conservación y Restauración y se valora la práctica, mediante un ejercicio. Sin embargo, no es posible contraponerlas y así se contemplará en la propia evolución de las enseñanzas de aquella originaria Escuela de Pintura y Escultura, después Escuelas y Facultades de Bellas Artes, contemplando sus planes de estudios de la misma fecha.

Entre la oposición de 1922 y la de 1969 media la Teoría de los Bienes Culturales, las transformaciones legislativas internacionales y nacionales, el nuevo concepto de Patrimonio Histórico, los nuevos conceptos y criterios de restauración, la propia creación del Instituto Central de Conservación y Restauración, pero fundamentalmente media el cambio cultural, legislativo y administrativo de la sociedad española en este final de siglo.

Un proceso histórico y cultural y no la simple relación o contraposición de centros docentes.

La contraposición de centros docentes, su disyuntiva y la doble vía de formación y titulación indica la existencia de un proceso puesto en marcha e inconcluso. Durante la década de los ochenta y terminado el proceso de reciclaje, los Restauradores se modelan por el nuevo perfil del Conservador Restaurador, trazado por la Escuela de Conservación y Restauración de Bienes Culturales y se crea un desconcierto general entre los profesionales, manifiesto en los congresos nacionales, abriéndose el debate sobre cualificación, formación y centros docentes, controversia que quedará simplemente anotada.

El análisis histórico aporta luz a estos acontecimientos demostrando ser una situación de tránsito, fruto y repercusión de un cambio profundo y lento. Esta es la idea reveladora nacida del análisis de la problemática del Conservador Restaurador a la luz del estudio histórico y cultural de los hechos.
(10) CABALLERO ZOREDA. L. FUnciones, Organización y Servicios de un Museo: Museo Arqueologico Nacional de Madrid.Asociación Española de Archiveros, Bibliotecarios, Museólogos y Documentalistas. Guadalajara, 1982, pág. 147.

(11) MORÓN DE CASTRO, Ma $\mathrm{F}$ "La situación actual del Restaurador en los Museos Andaluces", IX Congreso de Conservación y Restauración de Bienes Culturales, Sevilla, 1992.pág. 101

(12) Borrador del Programa que la Escuela de Pintura y Escultura propone para los ejercicios de oposición a la plaza de Restaurador vacante en el Museo Nacional. Original manuscrito sin datación. Encontrado en la Carpeta correspondiente a los documentos de 1922. Archivo de la Facultad de Bellas Artes de la Universidad Complutense de Madrid.ARAÑO GISBERT.J. C. "La Enseñanza de las Bellas Artes en España 18441980", Departamento de Didáctica y Organización Escolar Facultad de Ciencias de la Educación, Universidad de Sevilla, Apéndice documental C.7.

(13) MUÑOZ COSME, A. La Conservación del Patrimonio Arquitectónico Español, Ministerio de Cultura, Madrid, 1989, pág. 43.

(14) Orden de 20 de octubre de 1971 (Ministerio de Educación y Ciencia) (B.O.E. 4-12-1971), por la que se establece Examen de Reválida para Restauradores de Obras de Arte y Objetos Arqueológicos o Etnológicos que reúnan las circunstancias que se indican. Disposición $2{ }^{a}$.

(15) Orden de 13 de marzo de 1978 (Ministerio de Educación y Ciencia) (B.O.E. 22-3-1978), por el que se autoriza examen de conjun to de reválida a Restauradores de Obras de Arte en determinadas situaciones.

(16) La categoría Clase VI, D, fue dispuesta en 1959, (Decreto 1.639/1959, de 23 de septiembre (B.O.E. del 26-9-1959) y permanece vigente. 